\title{
Anterior Temporal Atrophy and Posterior Progression in Patients with Parkinson's Disease
}

\author{
Adriaan R.E. Potgieser ${ }^{a-c} \quad$ Anouk van der Hoorn ${ }^{a, b} \quad$ Anne Marthe Meppelink ${ }^{a, b}$ \\ Laura K. Teune $^{a, b}$ Janneke Koerts ${ }^{d}$ Bauke M. de Jong ${ }^{a, b}$ \\ ${ }^{a}$ Department of Neurology, ${ }^{b}$ Neuroimaging Center and ${ }^{\mathrm{c} D e p a r t m e n t}$ of Neurosurgery, University Medical Center \\ Groningen, and d Department of Clinical and Developmental Neuropsychology, University of Groningen, Groningen, \\ The Netherlands
}

\section{Key Words}

Parkinson's disease · Voxel-based morphometry · Atrophy

\begin{abstract}
Background: Parkinson's disease (PD) is characterized by specific motor and nonmotor impairments. This suggests that PD is characterized by disease-specific regional cortical atrophy. Given the change of symptoms over time, a concurrent increase in regional atrophy may further be assumed to reflect the dynamic process of disease progression. Methods: In this study we retrospectively collected T1-weighted MRI scans from previous studies performed in our center, enabling the comparison of gray matter atrophy in 77 PD patients with 87 controls using voxel-based morphometry (VBM). This large VBM analysis provided the opportunity to investigate cortical atrophy in relation with disease progression. Results: We found significant PD-related reductions of gray matter density bilaterally in the anterior temporal cortex, the left inferior frontal and left extrastriate visual cortex, independent from normal aging. The anterior temporal cortex did not show major progression, whereas particularly the posterior parts of the lateral temporal cortex and adjacent extrastriate visual cortex occurred at a later stage of disease. Conclusions: Temporal pole atrophy as an early sign of PD is
\end{abstract}

\section{KARGER}

E-Mail karger@karger.com

www.karger.com/ndd consistent with the PD pathology classification of Braak. The initial anterior temporal atrophy with spread to occipitotemporal and posterior parietal regions may subserve 'emotionbased' sensorimotor transformations and deficits in the visual domain, respectively, which may be regarded as premotor symptoms.

(c) 2014 S. Karger AG, Basel

\section{Introduction}

The main pathology in idiopathic Parkinson's disease (PD) is degeneration of the substantia nigra and its dopaminergic projections to the striatum [1], which is a major cause of the motor symptoms in this disease. This is associated with misfolding of $\alpha$-synuclein [2]. It is increasingly recognized, however, that PD not only involves the motor system, but inflicts deterioration in multiple domains. For instance, olfactory dysfunction is considered as a relatively early or even preclinical sign in PD [3], while in later stages pathological changes in olfaction-related brain areas have been described [4]. Cortical atrophy has been demonstrated to correlate with olfactory dysfunction in PD [5]. Especially in later stages, cognitive impairment, including visuospatial dysfunc- (c) 2014 S. Karger AG, Basel

$1660-2854 / 14 / 0143-0125 \$ 39.50 / 0$
A.R.E. Potgieser

Department of Neurology, University Medical Center Groningen

Hanzeplein 1, PO Box 30.001

NL-9700 RB Groningen (The Netherlands)

E-Mail a.r.e.potgieser@umcg.nl 
tion and alterations in executive functions, occurs relatively often in PD [6]. Such cortical dysfunction suggests that PD is characterized by a disease-specific regional atrophy. Given the change of symptoms over time, an associated change in the distribution of regional atrophy may further be assumed to reflect the dynamic process of disease progression. In a network view of the brain, it is reasonable to think that initial subcortical damage in PD may inflict cortical impairments in regions that receive projections from such subcortical sources [7]. One may thus hypothesize that long-term hypoactivation of the cortex by subcortical structures leads to cortical atrophy.

Voxel-based morphometry (VBM) studies in PD typically comprised small subject groups and yielded variable and even conflicting results [8]. In the present study, we retrospectively collected 3-tesla MRI images of $77 \mathrm{PD}$ patients that were included in previous studies performed at our center. This resulted in one of the largest VBM analyses comparing patients with PD and controls. Given the variation in disease duration of these patients, it also provided the opportunity to investigate cortical atrophy as an index of disease progression.

\section{Methods}

\section{Subjects}

T1-weighted images were retrospectively collected from all 7 studies performed in our center between 2007 and 2013 (104 controls, 111 PD patients). These studies concerned only $1 \mathrm{VBM}$ study [9] and 6 different functional MRI experiments that included the acquisition of a T1-weighted anatomical brain image used for coregistration and the assessment of general brain characteristics. Since some subjects participated in more than 1 study, we only used the last scan made in order to assess the patient scans with the most pronounced characteristics of disease. As $34 \mathrm{PD}$ patients and 8 controls participated in 2 or more studies, the control scans outnumbered the included PD scans. This provided the opportunity to make a selection of control subjects in order to get an optimally age-matched control group. To that end, we inspected the age distribution of both groups, after excluding the first of the possible double scans, and subsequently excluded 9 controls that were not in the same age group as the PD patients. This resulted in a total of 87 controls and 77 patients with PD who were included for further analysis. Patients met the criteria of the UK PD Society Brain Bank. Severity of motor symptoms was assessed using part III of the Unified Parkinson's Disease Rating Scale (UPDRS) [10]. The Mini-Mental State Examination (MMSE) was used as a screening tool for cognitive functions. An MMSE $\geq 24$ was considered to represent unimpaired cognition. Subjects suffering from neurological disorders other than PD or psychiatric disorders were excluded. All studies were approved by the local Medical Ethical Committee. Participants gave their written informed consent.

\section{Image Acquisition}

Data acquisition was performed using a 3-tesla Philips MR system (Best, the Netherlands) with a standard 8-channel SENSE head coil. In 3 of the 6 studies (49 controls, 45 PD patients) T1weighted 3 -dimensional anatomical scans were acquired with field of view $232 \times 256 \times 170, \mathrm{TR}=9.0 \mathrm{~ms}, \mathrm{TE}=3.5 \mathrm{~ms}$, flip angle $8^{\circ}$, 170 slices without slice gap and voxel size $0.9 \times 1 \times 1 \mathrm{~mm}$. The other 3 studies ( 38 controls, 32 PD patients) acquired T1-weighted scans with field of view $256 \times 160 \times 204 \mathrm{~mm}, \mathrm{TR}=25 \mathrm{~ms}, \mathrm{TE}=4.6$ $\mathrm{ms}$, flip angle $30^{\circ}, 160$ slices with slice gap -1 and voxel size $1 \times 1 \times$ $1 \mathrm{~mm}$. The two acquisition protocols were equally distributed over the groups.

\section{Image Analysis}

VBM is a method applied in MRI data sets, which offers the opportunity to compare the entire cortex across different groups without a priori assumptions on a subjective region of interest selection [11]. VBM enables the detection of differences in gray matter concentration on a local scale, while correcting for global shape differences [11]. Image processing and voxel-based statistical analysis were conducted using Statistical Parametric Mapping, version 8 (2009, Wellcome Department of Cognitive Neurology, London, UK; http://www.fil.ion.ucl.ac.uk/spm). Raw images were manually reoriented to put the middle of the anterior commissure at the coordinate $\mathrm{x} 0 . \mathrm{y} 0 . \mathrm{z} 0$ position. The images were processed using the unified segmentation approach with standard settings [12]. In this approach the images are segmented in gray matter, white matter and cerebrospinal fluid, bias corrected and spatially normalized to the Montreal Neurological Institute brain reference space, based on nonlinear registration with tissue probability maps. The segmented gray matter images were modulated and smoothed (full width at half maximum 8 ). We used the optimized VBM method in SPM8, because it is widely used and there is extensive experience with this method. An alternative might have been to use DARTEL. It has, however, been shown that DARTEL not necessarily results in better image registration in patients with Alzheimer's disease, frontotemporal dementia and semantic dementia, while it may be less sensitive than the method we used [13]. On the other hand, DARTEL has been reported to be more sensitive for detecting hippocampal changes in acute depression [14].

\section{Statistical Analysis of Behavioral Parameters}

MMSE scores and age were not normally distributed and therefore tested for differences between the groups with the MannWhitney $U$ test. Gender was compared using the $\chi^{2}$ test. A p value $<0.05$ was considered statistically significant.

\section{Image Statistical Analysis}

Total gray matter was calculated per subject and used as a covariate in the model to remove variance due to differences in brain size, because we were interested in regional gray matter differences. Also, the type of T1 scan was used as a covariate to correct for possible effects of differences in scanning parameters. Initial analysis assessed the presence of regional differences between patients and controls at voxel level $\mathrm{p}<0.001$ to see if there were any differences. Resulting clusters of regional difference were regarded statistically significant at $\mathrm{p}<0.05$ family-wise error (FWE) corrected with a cluster extent of 8 voxels. We compared healthy controls $(\mathrm{n}=87)$ with PD patients $(\mathrm{n}=77)$ on local gray matter density changes with ANOVA (flexible factorial design). To check wheth- 
Table 1. Subject characteristics

\begin{tabular}{|c|c|c|c|c|c|}
\hline & $\begin{array}{l}\text { Controls } \\
(\mathrm{n}=87)\end{array}$ & $\mathrm{n}$ & $\begin{array}{l}\mathrm{PD} \\
(\mathrm{n}=77)\end{array}$ & $\mathrm{n}$ & $\mathrm{p}$ value \\
\hline Age, years & $60.1(7.2)$ & 87 & $63.0(10.5)$ & 77 & 0.07 \\
\hline Gender, male & 50 & $85^{1}$ & 46 & $70^{1}$ & 0.42 \\
\hline MMSE (0-30) & $28.0(1.0)$ & $84^{1}$ & $27.0(1.3)^{2}$ & $70^{1}$ & 0.00 \\
\hline UPDRS III (0-56) & n.a. & n.a. & $24.3(7.5)$ & $69^{1}$ & n.a. \\
\hline $\begin{array}{l}\text { Disease duration, } \\
\text { years }\end{array}$ & n.a. & n.a. & $8.0(5.1)$ & $65^{1}$ & n.a. \\
\hline
\end{tabular}

Data presented as means with standard deviation in parentheses. n.a. = Not applicable.

${ }^{1}$ Lower subject number due to missing data.

${ }^{2}$ Score range $24-30$ in patients.

er the perceived atrophy was not merely the consequence of normal aging we similarly compared the 25 youngest controls with the 25 oldest controls. In addition, we compared the 87 healthy controls with the $25 \mathrm{PD}$ patients with the shortest duration of disease (PD_short) and the 25 patients with the longest disease duration (PD_long), respectively. These comparisons were expected to provide insight into disease progression. An additional comparison between the 25 PD_short and 25 PD_long patients complemented the two comparisons between patients and control subjects. The reason to introduce this binary split was to get a clear distinction between the subgroups with short and long disease duration, leaving out patients with intermediate disease duration. The large patient group indeed allowed such a selection.

\section{Results}

There were no significant differences between PD patients and healthy controls regarding age and gender, while the MMSE score was slightly but significantly lower in the patient group (table 1). None of the participants had an MMSE below 24.

\section{VBM of Patients with PD versus Controls}

Comparison of PD patients with healthy controls revealed significant reductions of regional gray matter density which was most pronounced in anterior temporal regions (fig. 1a; table 2). Adjacent to the cluster of left anterior temporal atrophy, inferior frontal atrophy was seen, while in the right hemisphere posterolateral frontal atrophy was seen at a more dorsal position. In posterior parts of the brain, cortical atrophy was identified in both ventrolateral and dorsal extrastriate visual areas of particularly the left hemisphere, together with significant right precuneus atrophy. At a relaxed threshold of $p<0.001$ voxel level uncorrected, the revealed pattern of reduced right occipital and parietal gray matter density was similar to that in the left hemisphere. There were no regions identified with more gray matter atrophy in controls compared to PD patients.

\section{Progression of Cortical Atrophy in Patients with PD}

Statistical analysis showed that disease duration of the 25 PD_short patients (mean duration 3.3 years, SD 1.2) was indeed significantly shorter compared to the $\mathrm{PD}_{-}$ long patients (mean 13.2 years, SD 4.1; $\mathrm{p}<0.001$ ). These two groups did not differ in age and MMSE. Mean age of PD_short was 61.8 years (SD 8.8) with a mean MMSE of 27.6 (SD 1.3) while it was 63.0 years (SD 7.1) for PD_long and MMSE 27.4 (SD 1.3) ( $\mathrm{p}=0.60$ for each parameter). Neither did the two groups differ in gender (15 males in PD_short and 16 males in PD_short, $\mathrm{p}=0.77)$. The groups had an equal distribution in the type of T1 scan (17 of the first sequence described in the Methods).

Compared to the control group, the 25 PD_short patients had cortical atrophy of both temporal poles ( $\mathrm{p}<$ 0.001 , voxel level uncorrected; fig. $1 \mathrm{~b}$ ). This difference in gray matter density did, however, not reach statistical significance at cluster level corrected for the entire brain volume (FWE). When the 25 PD_long patients were compared with the controls, significant gray matter reductions were seen at the two temporal poles and in left extrastriate cortical regions ( $p<0.05$, FWE corrected; fig. 1c).

Complementary to comparing the control subjects with successively the $\mathrm{PD}$ _short and $\mathrm{PD}$ _long patients, a direct comparison of PD_short with PD_long showed a progression of regional atrophy in the PD_long group at the lateral occipitotemporal junction, bilaterally, with a maximum in the right middle temporal gyrus ( $\mathrm{x} 54, \mathrm{y}-42$, $\mathrm{z}-4$; $\mathrm{p}<0.001$, voxel level uncorrected; fig. 1d; table 3). No differences were seen at the temporal poles. The clusters of advanced atrophy did, however, not reach significance at the cluster level corrected for the entire brain volume. This was also the case for changes in the right middle frontal gyrus (BA 45) and right supramarginal gyrus (BA 2).

\section{Comparison of Old and Young Controls}

In comparison with the 25 youngest controls (mean age 51.3 years, SD 4.3), the 25 oldest control subjects (mean age 68.1 years, SD 3.7) had a reduction of gray matter density in both the midcingulate cortex and superior surface of the left temporal lobe along the sylvian fissure (online suppl. fig. 1; for all online suppl. material, see www.karger.com/doi/10.1159/000363245). This differ- 

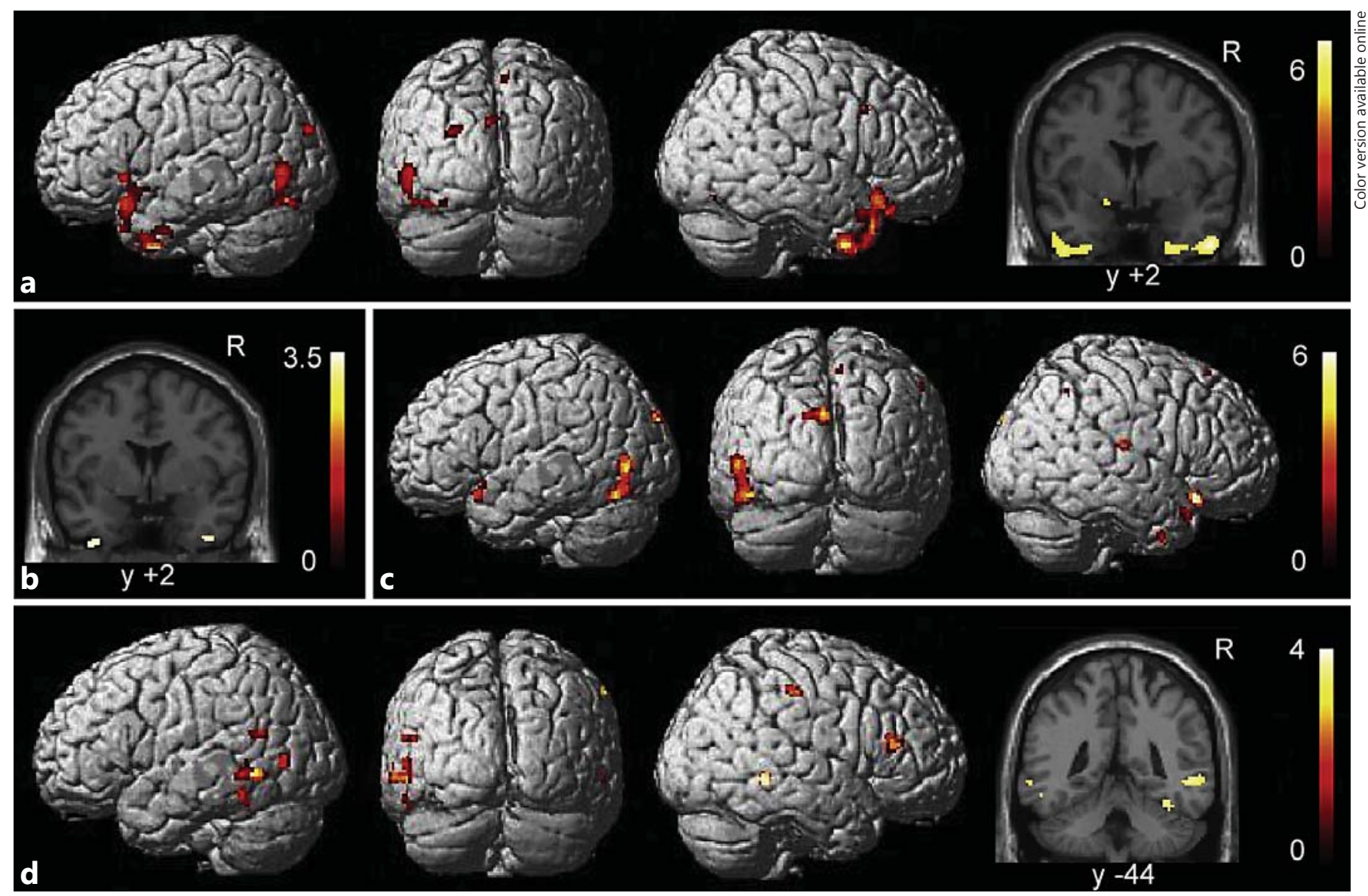

Fig. 1. a Clusters of significant regional reduction of gray matter density in patients with PD when compared to control subjects ( $\mathrm{p}<0.05$, FWE corrected, voxel extent $\mathrm{k}$ of 8 voxels). In addition to the brain volume images, the atrophy at the ventral surface of the temporal poles is depicted on a coronal slice at $y+2 \mathrm{~mm}$ relative to the vertical traversing the anterior commissure. $\mathbf{b}$ The single regions with reduction of gray matter density in the 25 patients with $\mathrm{PD}$ with the shortest disease duration compared to the 87 healthy control subjects ( $\mathrm{p}<0.001$, uncorrected, $\mathrm{k}$ of 8 voxels) in a coronal section $(y+2 \mathrm{~mm})$. c Regional reduction of gray matter density in the $25 \mathrm{PD}$ patients with the longest disease duration

ence in gray matter density only reached a subthreshold significance level of $\mathrm{p}<0.001$ (voxel level, uncorrected) while the difference in age was indeed statistically significant $(\mathrm{p}<0.01)$.

\section{Discussion}

In this large study with 77 PD patients, we found significant PD-related reductions of gray matter density in predominantly the anterior temporal cortex, bilaterally, compared to healthy controls ( $\mathrm{p}<0.05$, FWE corrected, $\mathrm{k}$ of 8 voxels). d Regional reduction of gray matter density of the $25 \mathrm{PD}$ patients with the longest disease duration when compared to the 25 patients with the shortest disease duration (voxel level $\mathrm{p}<0.001$, uncorrected, $\mathrm{k}$ of 20 voxels). In addition, the atrophy of the right fusiform gyrus, right middle temporal gyrus and left inferior temporal gyrus are depicted on a coronal slice at $\mathrm{y}-44 \mathrm{~mm}$ relative to the vertical traversing the anterior commissure. Clusters are rendered onto the volume or a section of a standard T1-weighted brain (Montreal Neurological Institute). $t$ values are indicated by the color bars; $\mathrm{R}=$ right side of the brain.

left inferior frontal and left extrastriate visual cortex. This distribution of regional atrophy was different from the observed aging-associated pattern of atrophy in temporal perisylvian and cingulate cortices. The latter was consistent with previous studies on normal aging [15]. Progression of atrophy in PD was inferred from the comparison between patients with the shortest and longest disease durations. This analysis indicated that the early anterior temporal atrophy did not show major progression, whereas atrophy of particularly the posterior parts of the lateral temporal cortex and adjacent ex- 
Table 2. Regional decreases in cerebral gray matter in patients with PD compared with controls

\begin{tabular}{|c|c|c|c|c|c|c|}
\hline Brain region & $\mathrm{x}$ & $\mathrm{y}$ & $\mathrm{z}$ & t value & Extent & p corrected \\
\hline \multicolumn{7}{|l|}{ Right hemisphere } \\
\hline Temporal pole (BA 38) & 32 & 14 & -28 & 6.65 & 979 & 0.000 \\
\hline Inferior temporal gyrus (BA 20) & 50 & 4 & -42 & 6.52 & s.c. & s.c. \\
\hline Fusiform gyrus (BA 19) & 26 & -70 & -14 & 5.47 & 18 & 0.012 \\
\hline Superior parietal lobule (BA 7) & 8 & -68 & 58 & 5.34 & 22 & 0.009 \\
\hline Rolandic operculum (BA 48) & 46 & -4 & 10 & 5.26 & 9 & 0.020 \\
\hline Dorsolateral prefrontal cortex (BA 46) & 52 & 16 & 36 & 5.48 & 15 & 0.014 \\
\hline \multicolumn{7}{|l|}{ Left hemisphere } \\
\hline Temporal pole (BA 38) & -30 & 18 & -32 & 5.40 & 253 & 0.000 \\
\hline Inferior frontal gyrus (BA 47) & -44 & 20 & -2 & 5.39 & s.c. & s.c. \\
\hline Inferior temporal gyrus (BA 20) & -56 & -6 & -34 & 5.46 & 260 & 0.000 \\
\hline Hippocampus (BA 27) & -24 & -10 & -12 & 5.49 & 75 & 0.001 \\
\hline Fusiform gyrus (BA 19) & -28 & -74 & -16 & 5.78 & 81 & 0.001 \\
\hline Anterior lingual gyrus (BA 27) & -12 & -36 & -6 & 5.74 & 36 & 0.005 \\
\hline Extrastriate visual cortex (BA 19) & -50 & -76 & 0 & 6.12 & 221 & 0.000 \\
\hline Superior occipital gyrus (BA 18) & -24 & -92 & 26 & 5.44 & 49 & 0.003 \\
\hline Cuneus (BA 18) & -2 & -84 & 34 & 5.21 & 31 & 0.006 \\
\hline
\end{tabular}

Coordinates (standard brain Montreal Neurological Insititute) refer to the voxels of maximum difference within clusters of significant difference ( $\mathrm{p}<0.05$ FWE corrected, voxel extent of 8 voxels). BA = Brodmann area; s.c. $=$ same cluster.

Table 3. Progression of cerebral gray matter loss in PD (PD_short > PD_long)

\begin{tabular}{lrrrrrl}
\hline Brain region & $\mathrm{x}$ & $\mathrm{y}$ & $\mathrm{z}$ & $\mathrm{t}$ value & Extent & p uncorrected \\
\hline Right hemisphere & & & & & & \\
Middle temporal gyrus (BA 21) & 54 & -42 & -4 & 3.9 & 56 & 0.24 \\
Fusiform gyrus (BA 19) & 36 & -40 & -20 & 4.3 & 82 & 0.16 \\
Supramarginal gyrus (BA 2) & 62 & -26 & 46 & 3.7 & 40 & 0.32 \\
Middle frontal gyrus (BA 45) & 60 & 32 & 16 & 3.8 & 64 & 0.21 \\
\hline Left hemisphere & & & & & & \\
Inferior temporal gyrus & -54 & -46 & -14 & 3.5 & 32 & 0.38 \\
Inferior temporal gyrus & -54 & -58 & -4 & 3.5 & 75 & 0.18 \\
Middle temporal gyrus (BA 21) & -56 & -56 & 20 & 3.3 & 21 & 0.48 \\
Extrastriate visual cortex (BA 19) & -54 & -72 & 4 & 3.5 & 28 & 0.41 \\
\hline
\end{tabular}

Coordinates (standard brain Montreal Neurological Institute, MNI) refer to voxels of maximal difference in clusters surviving a threshold of $\mathrm{p}<0.001$ uncorrected with a voxel extent of 20 voxels. BA $=$ Brodmann area.

trastriate visual cortex occurred at a later stage of disease, together with reductions of gray matter density in the right supramarginal and right inferior frontal gyrus. Temporal pole atrophy as an early sign of PD would seem consistent with the previous description of Braak et al. [4].

Progressive Atrophy in Parkinson's Disease

\section{Anterior Temporal Atrophy}

At first sight, atrophy at the temporal pole may seem at odds with the dominant characteristics of motor dysfunction, which define PD. On the other hand, the consistency of symptoms such as hyposmia that precede motor symptoms may point at an impaired function of 
the anterior (anteromedial) aspects of the temporal lobes $[3,5]$. Moreover, assessments of postmortem pathology in PD have indicated that, following intraneuronal brainstem pathology, initial cortical involvement particularly concerns the anteromedial temporal cortex [4]. Although particularly medial temporal atrophy in PD may hint at cognitive impairment [16], it has also been well documented for PD patients without signs of dementia [17]. It was intriguing to see that the most pronounced atrophy was found at the temporal pole, while progression of atrophy was not significantly present at this location. This may suggest that such a progression slows down in the course of disease. At an early disease stage, degeneration in particularly this region has indeed been shown by Braak et al. [4]. This might thus imply that due to advanced atrophy, additional progression is slower.

VBM examination of MR images for regional atrophy in PD patients, compared to healthy controls, has shown marked heterogeneity in the reported regions of atrophy, while some studies even failed to find any atrophy $[18,19]$. Relatively small study groups and differences in analysis methods are possible reasons for this variation. A common finding, nevertheless, appears to be atrophy of the temporal lobes, particularly in limbic and paralimbic regions [8]. However, with deformation-based morphometry there was only a significant contraction in the left cerebellum in early PD [20]. In a meta-analysis on clusters with significant gray matter reduction in PD obtained from 17 VBM studies involving 498 patients, Pan et al. [8] identified a single region of gray matter reduction comprising the left inferior frontal gyrus, anterior insula and left superior temporal gyrus [for a review in a broader perspective than VBM, see 21]. This focus of regional atrophy exactly corresponds to our main left-hemisphere cluster, while we found additional atrophy of the same region in the other hemisphere. Patient characteristics reported in the meta-analysis of Pan et al. [8] (mean age 66 years, disease duration 6.9 years and UPDRS III 24.1) indicated that our study represented an average PD population (mean age 63.0 years, disease duration 8.0 years and UPDRS III 24.3). Moreover, the variation in disease duration in our patient group with a mean of 3.3 years and 13.3 years for PD_short and PD_long, respectively, made it feasible to additionally examine the pattern of atrophy progression. The coherence of results from directly comparing $\mathrm{PD} \_$short with $\mathrm{PD}$ _long and from the comparison of controls with each of these PD subgroups laid ground for the conclusion that the initial anterior temporal atrophy particularly spread to occipitotemporal and posterior parietal regions.

The temporal pole is implicated in a wide range of functions, particularly characterized by the attribution of an emotional content to stimuli in various perceptual domains [22]. Such emotion-related processing not only concerns its perception, but is intrinsically related to emotional expression, including simple vegetative responses such as changed heart rate or releasing tears, as well as more complex motor behavior [23, 24]. In this respect, functional coherence between limbic system structures such as anteromedial temporal regions, adjacent insula, orbitofrontal cortex and ventral striatum is logically subserving 'emotion-based' sensorimotor transformations [23]. The amygdala complex, embedded anteromedially in the temporal lobe, seems to play an important role in the integration of internal emotional states and actual stimulus conditions in guiding e.g. the ventral striatum in the onset of behavioral responses [23, 25]. A functional consequence of coherent temporalstriatum involvement in such circuitry is that its dysfunction, associated with temporal pole atrophy, may not only lead to olfactory deficit, but also to PD premotor symptoms such as depression, anxiety and autonomic impairment [26]. In this respect, a typical PD symptom such as hypomimia may not only be regarded as a manifestation of motor dysfunction, but may well reflect a more complex impairment of emotional processing, including emotion-motor transformation. The role of the temporal pole in emotion perception has been well described [22]. For instance, it supports our empathizing with other people [27], while bilateral removal of the temporal poles in monkeys leads to a disturbance in recognizing, interpreting and reacting to social signals [28].

It is intriguing to notice that the left-lateralized frontotemporal atrophy in the referred meta-analysis of Pan et al. [8] fits the observation that PD-related deficit in the uncinate fascicle particularly occurred in the left hemisphere [29]. Although Kim et al. [29] described more widely distributed white matter changes in the PD brain, changes in the crucial connection between the temporal pole and orbitofrontal cortex underscore the anterior temporal involvement in PD pathology.

A biochemical relation between anteromedial temporal dysfunction and PD might be inferred from the dopamine innervation in normal human subjects, as revealed by ${ }^{18} \mathrm{~F}$ DOPA positron emission tomography. While striatum uptake of this tracer is the most evident feature of this functional imaging method, ${ }^{18} \mathrm{~F}-\mathrm{DOP} A$ uptake in limbic structures such as the entorhinal cortex and amygdala is more 
than 4 times higher than that of the neocortex of the occipital lobe [30]. This implies that impaired dopamine innervation from the upper brainstem may have a larger impact on the referred limbic than neocortical regions. This argument, however, was not supported by the assessment of extrastriatal uptake of this tracer, as limbic uptake was not specifically reduced in early PD stages [31].

\section{Posterior Cortical Atrophy}

In addition to anterior temporal atrophy, regional clusters of PD-related atrophy particularly comprised posterior temporal, extrastriate visual and posterior parietal cortical regions. Moreover, the progression of atrophy during disease took place in predominantly these posterior regions, which suggests that it might initially follow the course of the inferior longitudinal fasciculus [32] or, more stepwise, the functional ventral visual pathway in reversed direction [33]. Such a progression of atrophy towards posterior temporal and extrastriate visual cortical regions would be consistent with a specific PDrelated dysfunction in the visual domain. The latter includes hallucinations, which have been associated with impaired visual object processing in ventrolateral extrastriate visual areas [34]. The posterior parietal progression seems consistent with an important profile of neuropsychological dysfunction seen early in the course of the disease, which may predict cognitive decline [35]. The association between functional impairment of both visual and parietal cortex regions in PD [36] and observations that visual stimuli may have a stronger impact on gait control in this disease $[37,38]$ underscore that more knowledge on visual cortex changes is required for understanding distinct PD symptoms.

\section{Atrophy in Motor-Related Areas}

While prefrontal and parietal foci of atrophy were found in our study, it may seem quite remarkable that no atrophy was seen in the striatum and cortical regions more specifically involved in motor processing. In a network view of cerebral processing, one might logically expect that degeneration of a crucial node or hub, such as the substantia nigra pars compacta, would inflict changes in distant parts of such a network (e.g. the cortico/basal ganglia/thalamocortical loops). This view is supported by the distribution of decreased metabolism including the premotor cortex in patients with PD [36]. One may, in this respect, speculate that atrophy in these regions may occur at more advanced stages of $\mathrm{PD}$ following a preceding decrease in metabolism.

\section{Conclusion}

VBM in a large group of PD patients showed that cortical atrophy was most pronounced at the temporal poles and the occipitotemporal junction, while it spread toward the lateral temporal cortex and adjacent extrastriate cortex in advanced PD. This distribution of cortical pathology highlights the presence of 'premotor' symptoms in PD.

\section{Acknowledgments}

We thank K.S.F. Colman and C.M. Toxopeus for kindly providing their data from some of the included previous studies done at our center. This work was supported by a Junior Scientific Masterclass grant of the University of Groningen to A.H. and to A.R.E.P.

\section{Disclosure Statement}

A.H. received a sponsorship from Abbvie, L.K.T. and J.K. received grants from the International Parkinson Foundation (IPF).

\section{References}

Braak H, Braak E: Pathoanatomy of Parkinson's disease. J Neurol 2000;247(suppl 2):II3II10.

-2 Olanow CW, Brundin P: Parkinson's disease and alpha synuclein: is Parkinson's disease a prion-like disorder? Mov Disord 2013;28:3140.

- 3 Ponsen MM, Stoffers D, Booij J, van Eck-Smit BL, Wolters EC, Berendse HW: Idiopathic hyposmia as a preclinical sign of Parkinson's disease. Ann Neurol 2004;56:173-181.
Braak H, Del Tredici K, Rub U, de Vos RA, Jansen Steur EN, Braak E: Staging of brain pathology related to sporadic Parkinson's disease. Neurobiol Aging 2003;24: 197-211.

5 Wu X, Yu C, Fan F, Zhang K, Zhu C, Wu T, et al: Correlation between progressive changes in piriform cortex and olfactory performance in early Parkinson's disease. Eur Neurol 2011;66:98-105.
Caballol N, Marti MJ, Tolosa E: Cognitive dysfunction and dementia in Parkinson disease. Mov Disord 2007;22(suppl 17):S358-S366.

7 Alexander GE, DeLong MR, Strick PL: Parallel organization of functionally segregated circuits linking basal ganglia and cortex. Annu Rev Neurosci 1986;9:357-381.

-8 Pan PL, Song W, Shang HF: Voxel-wise metaanalysis of gray matter abnormalities in idiopathic Parkinson's disease. Eur J Neurol 2012; 19:199-206. 
D9 Meppelink AM, de Jong BM, Teune LK, van Laar T: Regional cortical grey matter loss in Parkinson's disease without dementia is independent from visual hallucinations. Mov Disord 2011;26:142-147.

10 Fahn S, Elton RL: Unified Parkinson's disease rating scale; in Fahn S, Marsden CD, Calne D, Goldstein M (eds): Recent Developments in Parkinson's Disease. Florham Park, Macmillan Health Care Information, 1987, pp 153163.

-11 Ashburner J, Friston KJ: Voxel-based morphometry - the methods. Neuroimage 2000; 11:805-821.

12 Ashburner J, Friston KJ: Unified segmentation. Neuroimage 2005;26:839-851.

$>13$ Pereira JM, Xiong L, Acosta-Cabronero J, Pengas G, Williams GB, Nestor PJ: Registration accuracy for VBM studies varies according to region and degenerative disease grouping. Neuroimage 2010;49:2205-2215.

-14 Bergouignan L, Chupin M, Czechowska Y, Kinkingnéhun S, Lemogne C, Le Bastard G, Lepage M, Garnero L, Colliot O, Fossati P: Can voxel based morphometry, manual segmentation and automated segmentation equally detect hippocampal volume differences in acute depression? Neuroimage 2009; 45:29-37.

15 Good CD, Johnsrude IS, Ashburner J, Henson RN, Friston KJ, Frackowiak RS: A voxel-based morphometric study of ageing in 465 normal adult human brains. Neuroimage 2001;14: 21-36.

$>16$ Laakso MP, Partanen K, Riekkinen P, Lehtovirta M, Helkala EL, Hallikainen M, et al: Hippocampal volumes in Alzheimer's disease, Parkinson's disease with and without dementia, and in vascular dementia: an MRI study. Neurology 1996;46:678-681.

-17 Tam CW, Burton EJ, McKeith IG, Burn DJ, O'Brien JT: Temporal lobe atrophy on MRI in Parkinson disease with dementia: a comparison with Alzheimer disease and dementia with Lewy bodies. Neurology 2005;64:861865.
18 Dalaker TO, Zivadinov R, Larsen JP, Beyer MK, Cox JL, Alves G, et al: Gray matter correlations of cognition in incident Parkinson's disease. Mov Disord 2010;25:629-633.

-19 Feldmann A, Illes Z, Kosztolanyi P, Illes E, Mike A, Kover F, et al: Morphometric changes of gray matter in Parkinson's disease with depression: a voxel-based morphometry study. Mov Disord 2008;23:42-46.

20 Borghammer P, Østergaard K, Cumming P, Gjedde A, Rodell A, Hall N, Chakravarty MM: A deformation-based morphometry study of patients with early-stage Parkinson's disease. Eur J Neurol 2010;17:314-320.

21 Dagher A, Nagano-Saito A: Functional and anatomical magnetic resonance imaging in Parkinson's disease. Mol Imaging Biol 2007;9: 234-242.

22 Olson IR, Plotzker A, Ezzyat Y: The enigmatic temporal pole: a review of findings on social and emotional processing. Brain 2007;130: 1718-1731.

23 Mogenson GJ, Jones DL, Yim CY: From motivation to action: functional interface between the limbic system and the motor system. Prog Neurobiol 1980;14:69-97.

24 Gloor P, Olivier A, Quesney LF, Andermann F, Horowitz S: The role of the limbic system in experiential phenomena of temporal lobe epilepsy. Ann Neurol 1982;12:129-144.

25 Cardinal RN, Parkinson JA, Hall J, Everitt BJ: Emotion and motivation: the role of the amygdala, ventral striatum, and prefrontal cortex. Neurosci Biobehav Rev 2002;26:321352.

26 Siderowf A, Lang AE: Premotor Parkinson's disease: concepts and definitions. Mov Disord 2012;27:608-616.

27 Carr L, Iacoboni M, Dubeau MC, Mazziotta JC, Lenzi GL: Neural mechanisms of empathy in humans: a relay from neural systems for imitation to limbic areas. Proc Natl Acad Sci USA 2003; 100:5497-5502.
28 Kling A, Steklis HD: A neural substrate for affiliative behavior in nonhuman primates. Brain Behav Evol 1976;13:216-238.

$\checkmark 29$ Kim HJ, Kim SJ, Kim HS, Choi CG, Kim N, Han S, et al: Alterations of mean diffusivity in brain white matter and deep gray matter in Parkinson's disease. Neurosci Lett 2013;550: 64-68.

$>30$ Moore RY, Whone AL, McGowan S, Brooks DJ: Monoamine neuron innervation of the normal human brain: an ${ }^{18} \mathrm{~F}-\mathrm{DOPA}$ PET study. Brain Res 2003;982:137-145.

31 Moore RY, Whone AL, Brooks DJ: Extrastriatal monoamine neuron function in Parkinson's disease: an ${ }^{18} \mathrm{~F}$-dopa PET study. Neurobiol Dis 2008;29:381-390.

32 Catani M, Jones DK, Donato R, Ffytche DH: Occipito-temporal connections in the human brain. Brain 2003;126:2093-2107.

33 Kravitz DJ, Saleem KS, Baker CI, Ungerleider LG, Mishkin M: The ventral visual pathway: an expanded neural framework for the processing of object quality. Trends Cogn Sci 2013;17:26-49.

34 Meppelink AM, de Jong BM, Renken R, Leenders KL, Cornelissen FW, van Laar T: Impaired visual processing preceding image recognition in Parkinson's disease patients with visual hallucinations. Brain 2009;132:29802993.

35 Williams-Gray CH, Foltynie T, Brayne CE, Robbins TW, Barker RA: Evolution of cognitive dysfunction in an incident Parkinson's disease cohort. Brain 2007;130:1787-1798.

-36 Teune LK, Bartels AL, de Jong BM, Willemsen AT, Eshuis SA, de Vries JJ, et al: Typical cerebral metabolic patterns in neurodegenerative brain diseases. Mov Disord 2010;25: 2395-2404.

37 Schubert M, Prokop T, Brocke F, Berger W: Visual kinesthesia and locomotion in Parkinson's disease. Mov Disord 2005;20:141-150.

38 Van der Hoorn A, Hof AL, Leenders KL, de Jong BM: Narrowing wide-field optic flow affects treadmill gait in left-sided Parkinson's disease. Mov Disord 2012;27:580-581. 